\title{
BOTULISM AS A FOOD POISONING: WHAT IS IT?
}

\section{By}

MAMDOUH M. EL-BAHNASAWY ${ }^{1}$, NAGWA ZEIN-EL-ABDEEN ALY ${ }^{2}$, MAGDA ABDEL HAMEED ABDEL-FATTAH ${ }^{3}$ AND TOSSON A. MORSY ${ }^{4}$ Military Medical Academy ${ }^{1,2}$, Department of Nursing Administration ${ }^{3}$, Faculty of Nursing, Cairo University, and Department of Parasitology ${ }^{4}$, Faculty of Medicine, Ain Shams University, Cairo 11566, Egypt

\begin{abstract}
Botulism is a rare but potentially life-threatening neuroparalytic syndrome resulting from the action of a neurotoxin elaborated by the microorganism Clostridium botulinum. This disease has a lengthy history; the first investigation of botulism occurred in the 1820s with a case report on hundreds of patients with "sausage poisoning" in a southern German town. Several decades later in Belgium, the association was demonstrated between a neuromuscular paralysis and ham infected by a spore forming bacillus that was isolated from the ham. The organism was named Bacillus botulinus after the Latin word for sausage, botulus.
\end{abstract}

Key words: Botulism, Travellers, Egypt.

\section{Review and Discussion}

The modern syndrome of botulism occurs in five forms, differentiated by the mode of acquisition: Food-borne botulism-ingestion of food contaminated by preformed botulinum toxin. Infant botulism-the ingestion of clostridial spores that then colonize the host's gastrointestinal (GI) tract and release toxin produced in vivo Wound botulism infection of a wound by $C$. botulinum with subsequent in vivo production of neurotoxin Adult enteric infectious botulism or adult infectious botulism of unknown sourcesimilar to infant botulism in that toxin is produced in vivo in the GI tract of an infected adult host. The inhalational botulism-the form that would occur if aerosolized toxin was released in an act of bioterrorism (Abrutyn, 1998). Botulism has become the subject of the speculation as a possible bioterrorism threat.

Characteristics: $C$. botulinum is actually a heterogeneous group of gram-positive, rodshaped, spore forming, obligate anaerobic bacteria. They are ubiquitous, easily isolated from the surfaces of vegetables, fruits, seafood, and in soil and marine sediment worldwide. Eight strains of $C$. botulinum have been distinguished based upon the antigenic specificities of their toxins; a single strain almost always produces only one toxin type.

Spores of $C$. botulinum are heat-resistant, easily surviving $100^{\circ} \mathrm{C}$ at one atmosphere for five or more hours. However, spores can be destroyed by heating to $120^{\circ} \mathrm{C}$ for five minutes. When appropriate environmental conditions are present, spores will germinate and grow into toxin-producing bacilli. These environmental parameters include: The restricted oxygen exposure (either an anaerobic or semi-anaerobic environment), the low acidity $(\mathrm{pH}>4.6)$ water A temperature of 25 $37^{\circ} \mathrm{C}$ for ideal growth; however, some strains may grow in temperatures as low as $4^{\circ} \mathrm{C}$ (Dowell, 1984).

Some strains (A \& B) produce proteolytic enzymes that denature and "spoil" the foods they inhabit, leaving them with an unpleasant appearance, taste or smell. Other strains do not overtly change the food, and thus, contamination cannot reliably be suspected on the basis of the look, odor, or taste of the food.

Eight distinct $C$. botulinum toxin types have been described: A, B, C1, C2, D, E, F, and $\mathrm{G}$. Of them, types $\mathrm{A}, \mathrm{B}, \mathrm{E}$, and rarely $\mathrm{F}$ and $G$ cause human disease, while $C$ and $D$ cause disease in animals such as cattle, ducks, and chickens. Clostridium butyricum 
and C. barati are two other distinct Clostridia species that are known to have produced botulinum type $\mathrm{E}$ and $\mathrm{F}$ toxins. In contrast to the spores, the toxin is a heat-labile polypeptide readily denatured by heating above $80^{\circ} \mathrm{C}$. The polypeptide toxin is composed of a light and heavy chain with a combined molecular weight of 150 to $165 \mathrm{kDa}$ (Aureli et al, 1986).

Regardless of the route of entry into the body, the toxin disperses widely via vascular system and binds to a specific receptor (synaptotagmin II) on the presynaptic sides of peripheral cholinergic synapses at ganglia and neuromuscular junctions. The heavy chain of the toxin binds to the receptors, allowing the light chain to translocate into the nerve cell via the receptor mediated endocytosis.

After gaining entrance to the cell's cytoplasm, the toxin produces an irreversible disruption in stimulation-induced acetylcholine release by that presynaptic nerve terminal. Return of synaptic function requires sprouting of a new presynaptic terminal with subsequent formation of a new synapse, a process that requires approximately six months. Adrenergic synapses are not affected by the toxin, nor does the toxin appear to permeate the blood brain barrier, therefore limiting involvement to peripheral cholinergic nervous system (Jin et al, 2006).

Botulinum toxin is the most potent bacterial toxin, and perhaps the most potent known poison. The MLD (minimum lethal dose in experimental mice) of botulinum toxin is $0.0003 \mathrm{mcg} / \mathrm{kg}$. By comparison, the MLDs for curare and sodium cyanide are 500 and $10,000 \mathrm{mcg} / \mathrm{kg}$, respectively. It is estimated that one gram of aerosolized botulism toxin could kill at least 1.5 million people.

The toxin itself has no smell or taste. If ingested, the toxin is primarily absorbed by the stomach and small intestine, although the large intestine is capable of absorbing the toxin as well. The toxin is resistant to degradation by gastric acidity and human alimen- tary enzymes alike. However, botulinum toxin is inactivated in chlorinated water after only twenty minutes of exposure and in fresh water after three to six days (Pickett et al, 1976).

Incidence of cases by mode of acquisition: An average of 110 cases of botulism was reported each year in the United States according to the CDC. Approximately $72 \%$ of these cases are infant botulism, 25\% are food-borne botulism, and the remaining 3\% wound botulism. Adult infectious botulism is only occasionally reported.

Infant botulism has been recognized as a separate disease entity since 1976 . Since that time, over 900 cases of infant botulism have been reported in the United States; making it the most frequently reported form of botulism in the world. Despite the frequency of infant botulism among all cases of botulism, the CDC noted little change in the number of cases of infant or food-borne botulism in recent years (Werner et al, 2000).

By contrast, reports of wound botulism have increased dramatically, most noticeably in California. From 1951 through 1987, an average of 0.49 wound botulism cases per year were reported to California Department of Health Services compared to 2.25 cases per year in 1988 through 1991, three cases in 1992, four in 1993, 11 in 1994, and 23 in 1995.

A new cause, iatrogenic botulism, occurred in four patients; who received botulinum toxin injections using an unlicensed, the highly concentrated preparation of botulin-um toxin A. The patients may have received doses as high as 2857 times the estimated the human lethal dose by injection and had serum toxin levels 21 to 43 times the estimated lethal human dose by injection. After administration of equine serum anti-toxin, all patients survived but required prolonged mechanical ventilation and physical rehabilitation (Chertow et al, 2006).

Sources of the organism: 1- Wounds: Vast majority of cases of wound botulism in California since 1988 have involved injection 
drug users, particularly those using "black tar" heroin by the subcutaneous or the intramuscular (not intravascular) route. How the heroin becomes contaminated with the clostridial organisms or their toxins is unknown. Wound botulism has also been reported in patients who inhale cocaine. The patients presented with sinusitis, with one patient growing $C$. botulinum from a sinus aspirate (Roblot et al, 2006).

2- Food-borne botulism: Food-borne cases of botulism are most commonly recognized as small outbreaks, usually involving home canned foods such as fruits, vegetables, and fish. Commercial products and restaurants are occasionally sources (Sheth et al, 2008). The highest rates of food-borne botulism in the United States occur in Alaska as a result of ingestion of fermented fish. Surveillance data from the Centers for Disease Control and Prevention between 1990 and 2000 found that 103 of 263 (39\%) cases occurred in Alaska. Toxin types A, B, and E have all been associated with food-borne botulism (Townes et al, 1996).

A large outbreak of botulism involving 209 persons occurred in Thailand in March 2006 related to home-canned bamboo shoots. Ill persons had attended an annual religious right where food was consumed and home-canned bamboo shoots were the only item eaten by all of the affected persons. C. botulinum was subsequently cultured from leftover bamboo shoots. Of the 209 persons affected, 141 were hospitalized and 42 required the mechanical ventilation (Kongsaengdao et al, 2006).

Commercial carrot juice was identified as a source of type A botulism that resulted in the hospitalization of six patients in Georgia, Florida and Ontario, Canada, in September 2006. Improper refrigeration during transport or storage was suspected to be responsible for growth of $C$. botulinum contaminating the juice, allowing production of botulinum toxin. In addition, no preservatives, salt, or sugars were added to the carrot juice, which may be necessary to prevent the ger- mination of naturally present $C$. botulinum spores in the event of improper refrigeration.

3- Infant botulism: Infant botulism has long been associated with the ingestion of raw honey. However, this was most likely a minor environmental reservoir, since widespread education of the public has done little to affect the incidence of infant botulism in the United States. Most cases in there were thought to result from ingestion of environmental dust containing C. botulinum spores. The incidence of reported cases is highest in Utah, Pennsylvania, and California, states in which soil botulinum spore counts are high. Infant botulism results from intestinal colonization by $C$. botulinum and affects infants between one week and 12 months of age. Most cases occurred between two and eight months of age, and the median age of onset was three to four months. Cases presentation and severity are variable, most likely due to variations in the bacterial inoculum size and in host susceptibility. The initial presentation might involve constipation, shortly followed by weakness, feeding difficulties, descending or global hypotonia, drooling, anorexia, irritability, and weak cry (Barnes et al, 2013).

Botulism is classically described as the acute onset of bilateral cranial neuropathies associated with the symmetric descending weakness. The CDC has also suggested that the following be considered as key features of the botulism syndrome: The absence of fever Symmetric neurologic deficits. The patient remains responsive Normal or slow heart rate and normal blood pressure No sensory deficits with the exception of blurred vision.

The nonspecific gastrointestinal symptoms also may occur and occasionally predominant manifestations (Sobel et al, 2007).

4- Food-borne botulism: The onset of symptoms in food-borne botulism usually begins within 12 to 36 hours after ingestion of the preformed toxin, but the incubation period may range from several hours to one week. Prodromal symptoms often include 
nausea, vomiting, abdominal pain, diarrhea, and dry mouth with sore throat, but these symptoms can occur at any time during the course of illness (Hughes et al, 1981).

The cranial nerve involvement most commonly marks the onset of symptomatic illness and can include blurred vision (secondary to fixed pupillary dilation and palsies of cranial nerves III, IV, \& VI), diplopia, nystagmus, ptosis, dysphagia, dysarthria, and facial weakness. The descending muscle weakness usually progresses to the trunk and upper extremities, followed by the lower extremities. Urinary retention and constipation are common resulting from smooth muscle paralysis. But, occasionally paresthesias and asymmetric limb weakness are seen. Similar to infant botulism, respiratory difficulties (dyspnea) requiring the intubation and the mechanical ventilation are common caused by the diaphragmatic paralysis, upper airway compromise, or by both. Despite the evidence of neurologic involvement, cerebrospinal fluid analysis is normal (Mechem and Walter, 1994).

The disease presentation can be quite variable, ranging from mild complaints or to death within the first 24 hours of developing symptoms. A small outbreak of clinically mild botulism resulted from exposure to low doses of botulinum toxin type $\mathrm{E}$ from the contaminated, home-salted fish. All five patients presented with the gastrointestinal symptoms and two had minimal cranial nerve palsies. In other mild cases, ocular cranial nerve involvement is minimal or the sole manifestation of the illness.

In wound botulism theory, the botulism should only be associated with puncture wounds, subcutaneous abscesses, and deep space infections, which provide the anaerobic environment required for spores to germinate and the organism to thrive. However, cases have been described involving abrasions, lacerations, open fractures, surgical incisions, and even the closed hematoma without the appreciable skin defect.
Wound botulism differs only slightly from food-borne botulism in presentation. Wound botulism lacks the prodromal GI symptoms common to food-borne botulism and has a longer incubation period of approximately ten days. The patients may also have fever with wound botulism, but this probably results from concurrent bacterial infection of the wound by non-clostridial species.

Adult infectious botulism-Patients with this form of botulism develop enteric colonization with Clostridia and are found to have spores in their feces or in ingested food products. No preformed toxin is identified in any food source. The normal GI tract, except in infants, is resistant to colonization by $C$. botulinum. The changes in intestinal flora or mucosal defense required to allow this colonization are not known. However, it is thought that the achlorhydria, GI diseases, or the post-operative state predispose to colonization. This syndrome resembles infant botulism in its symptoms and course.

Diagnosis: Differential diagnosis for the food-borne, wound, and adult enteric botulism includes: myasthenia gravis, LambertEaton myasthenic syndrome (LEMS), tick paralysis, Guillain-Barré syndrome, poliomyelitis, stroke, and heavy metal intoxication. Less likely diagnoses include tetrodotoxin and shellfish poisoning and antimicrobial-associated paralysis.

Myasthenia gravis lacks autonomic symptoms, while these symptoms, especially invo-lving the salivation and erectile function, are common in LEMS; both the entities can be excluded by electromyogramphy or antibody study. Tensilon (edrophonium) tests to rule out myasthenia gravis should not be conducted, as they are often falsely positive in patients with botulism. Guillain-Barré syndrome usually involves ascending paralysis, sensory findings, and elevated cerebrospinal fluid protein. A tick check should be performed as part of the physical examination since the ticks causing tick paralysis may still be attached when the patient presents (Mosabah and Morsy, 2012). Cerebro- 
spinal fluid (CSF) studies of most patients with meningitis and encephalitis would reveal elevated protein and red and white blood cells. White blood cell counts, CSF studies, and sedimentation rates are all normal in patients with botulism. The most important issue in the diagnosis of any of the four syndromes of botulism is initial consideration of the disease. A careful his-tory and physical examination are essential.

Infant botulism is a rare disorder and the diagnosis is often missed. As an example, the diagnosis was suspected on admission in only one-half of infants enrolled in a randomized trial testing the efficacy of botulinum immune globulin.

Serum assays for botulinum toxin are often negative in cases of infant botulism. The diagnosis is supported by the isolation of $C$. botulinum spores from the stool and is confirmed by the identification of botulinum toxin in stool samples. However, the stool sample assays do not yield timely results. Anaerobic cultures often take up to six days for growth and identification of the causative organism and initial detection of toxin requires one to four days.

The delays with these confirmatory tests are important since therapy should be administered as early as possible. Thus, a presumptive diagnosis should be made based upon the clinical presentation and electrophysiologic findings, while the confirmatory stool studies are pending (Arnon et al, 2006).

Food-borne botulism: Patients presenting with clinical signs and symptoms of botulism should have serum analysis for toxin by bioassay in mice. Demonstration of toxin in the blood is diagnostic. This laboratory test was done by the special laboratories whose locations might be obtained by contacting state epidemiologists or regional offices of the CDC (1992).

Analysis of stool, vomitus, and suspected food items may also reveal toxin, which is diagnostic when coupled with the appropriate clinical and neurologic findings. EMG studies may be useful in these patients as well, but are not generally required.

Wound botulism: As with food-borne and infant botulism, the history and physical examination are keys to the diagnosis. The history should include questions about injection drug use and trauma. The only type of botulism to commonly involve fever and leukocytosis is wound botulism. One study found nearly half of the patients examined to have leukocytosis during their clinical course.

EMG is a useful diagnostic tool in wound as well as in infant botulism. Isolation of $C$. botulinum from the wound site is diagnostic and should be attempted. Stool and vomitus assays for toxin yield nothing in cases of wound botulism and should not be attempted. Serum assays for toxin are almost negative and therefore, being rarely helpful (O'Suilleabhain et al, 1998).

Treatment: Any patient with clinical signs, symptoms, or history suspicious for botulism should be hospitalized immediately and meticulously monitored for signs of the respiratory failure.

Monitoring should include pulse Oximetry, Spirometry, arterial blood gas measurement, and clinical evaluation of ventilation, per-fusion, and upper airway integrity. Respiratory failure is the primary cause of death in these patients. Prompt intubation with mechanical ventilation would dramatically decrease the risk of mortality.

Intubation should be considered for those patients with inadequate or worsening upper airway competency, and those with a vital capacity less than $30 \%$ of predicted. Infants and severe adult cases may require the prolonged mechanical ventilation. The supportive care in these cases should also include small volume continuous nasogastric feedings, to minimize aspiration risk. When severe ileus is present, parenteral hyperalimentation may be required.

There are two botulism antitoxin therapies available in the United States. Equine serum trivalent botulism antitoxin is used to treat children older than one year of age and 
adults; human-derived botulism immune globulin is used for infants less than one year of age.

Antitoxin therapy with equine serum trivalent botulism antitoxin (A, B, E) is available in the United States through State Health Departments or CDC Director's Emergency Operations Center should be contacted 770-488-7100 in USA (Tacket et al, 1984).

One retrospective review of 132 patients with type A food-borne botulism showed that antitoxin therapy decreased the fatality rates compared to untreated patients. The antitoxin treatment initiated after 24 hours of symptom onset did not shorten the symptoms duration, but did decrease fatality rates (Rossetto et al, 2013).

In the outbreak in Thailand related to the contaminated bamboo shoots, patients who received antitoxin on day four compared to those who received treatment on day six had a significantly reduced duration of mechanical ventilation (Schreiner et al, 1991).

If the clinical suspicion for botulism was high and symptoms were progressing, the administration of antitoxin should not be delayed for serum, stool, or wound study. The trivalent antitoxin eliminates the need to await toxin typing results. Because antitoxin is derived from horse serum, anaphylaxis and serum sickness are observed. Past reviews have reported incidences of $20 \%$ for serum sickness and 3\% for anaphylaxis. Guidelines for skin testing, desensitization, and dosing are all included in the antitoxin package (Sellin, 1984).

Human-derived botulinum immune globulin (called BIG-IV or Baby-BIG) is available for intravenous use in infants less than one year of age who are diagnosed with infant botulism. BIG-IV should be administered as early as possible in the illness.

Antibiotic therapy is unproven by clinical trial, but widely used and recommended for wound botulism after antitoxin has been administered. Penicillin G (3 million units IV every four hours in adults) provides ef- fective coverage of other clostridial species and is frequently used. The metronidazole (500 mg IV every eight hours) is a possible alternative for penicillin-allergic patients. Because of the risk of polymicrobial infection in cases of wound botulism, patients with this form of the disease should promptly receive antimicrobial therapy, especially when presented with the leukocytosis, fever, an abscess, or cellulitis. Therapy may need to be broadened in wound botulism to cover other organisms recovered from the wound. However, the use of the aminoglycosides is the contra-indicated, since they have been reported to induce neuro-muscular blockade, potentiating the effects of the toxin in these patients (Santos et al, 1981).

Antibiotics are not recommended for infant botulism or for adults with the suspected gastrointestinal botulism because lysis of intraluminal $C$. botulinum could increase the amount of toxin available for absorption (Reddy and Bleck, 2010).

Other treatment: In food-borne botulism, laxatives, enemas, or other cathartics may be given, provided no significant ileus is present. Patients presenting with wound botulism should undergo the extensive debridement, even if the wound appears unimpressive. These patients should receive tetanus boosters as well if it has been five or more years since their last immunization.

Botulism of any type usually requires hospitalization for one to three months. With prompt attention to impending respiratory failure and supportive care, mortality in botulism ranges from less than $5 \%$ to $8 \%$ (including infants). Mortality rate for infant botulism, the most common form in the United States, is less than 1\% (Simpson, 2013).

A retrospective review of 706 patients hospitalized for food-borne botulism in the Republic of Georgia, which has the highest reported rate of foodborne botulism in the world, suggested that the prognosis can be estimated based upon the manifestations of the disease: Shortness of breath alone might 
identify patients at increased mortality risk (18 versus $1 \%$ without this symptom). In addition, shortness of breath was present in 50 of 54 patients who died. In comparison, there were no deaths among 209 patients without shortness of breath, facial muscle weakness, or vomiting (Radšel et al, 2013).

Pifko et al. (2014) reported that infant botulism leads to a life-threatening descen-ding motor weakness and flaccid paralysis with symptoms such as constipation, weak-ness, and hypotonia and could lead to respiratory failure. Botulism immune globulin (BIG) was created to treat this deadly disease and functions by neutralizing all systemically circulating botulism toxins. They declared that in children with clinically diagnosed infant botulism, before diagnostic confirmation, and led to a significant reduction in intensive care unit and hospital stay for these patients.

The prospective validation of these observations is necessary. Overall, most patients with prompt hospitalization and respiratory care can expect a complete or nearly complete recovery with return to previous level of functioning. Long-term morbidity is low in patients with mild disease, with complete resolution of symptoms generally occurring within the first three months. In comparison, patients with severe disease may experience protracted courses involving years of the neurologic deficits, sequelae from extended mechanical ventilation, and nosocomial illness.

The largest reported experience on the long-term outcomes after acute paralytic botulism comes from a case-control study of 217 such patients in the Republic of Georgia and three randomly selected community controls for each patient (Gottlieb et al, 2007). During the initial infection, 15 had been hospitalized for at least one month, and $25 \%$ required mechanical ventilation.

The following findings were noted at a median of 4.3 years in the 211 patients who were still alive: $68 \%$ (compared to $17 \%$ of controls, matched odds ratio 17.6) reported their current health as being worse than six years before the interview; and $49 \%$ rated their health as fair or poor (compared to $25 \%$ of controls, matched odds ratio 5.0 ).

Compared to controls, the patients were also significantly more likely to report the symptoms such as fatigue, weakness, dizzyness, difficulty breathing with moderate exertion, and impaired psychosocial wellbeing. The requirement for mechanical ventilation during acute illness and older age were independent predictors of worse longterm health.

\section{In Egypt:}

Hibbs et al. (1991) reported that during an outbreak of type E foodborne botulism in Cairo in 1991, an investigational equine $\mathrm{F}$ $(a b ') 2$ "despeciated" heptavalent botulism immune globulin (dBIG) was provided to the Egyptian Ministry of Health by the U.S. Army. Of 54 patients known to have been treated with antitoxins, four received commercially available trivalent antitoxins, 45 received $\mathrm{dBIG}$, and 5 received both commercial antitoxin and dBIG. Physicians recorded side effects in $10(22 \%)$ of 45 patients who received $\mathrm{dBIG}$; in nine cases, reactions were considered "mild," and in one case they were believed to be serum sick-ness. In contrast, possible serum sickness during hospitalization was recorded for two of four patients who were receiving the commercial antitoxins. No complications of therapy were noted for any patient who was receiving both antitoxin types. In a separate study, 31 patients were contacted about their reactions to the antitoxin by telephone after hospital discharge. Seven (54\%) out of 13 patients attributed symptoms that they experienced while they were hospitalized to receipt of dBIG, while four (44\%) of nine patients who indicated that they had received commercial antitoxins and one (20\%) of five who received both commercial antitoxin and dBIG reported side effects before discharge. Data on the efficacy of the antitoxins were not obtained. 
Weber et al. (1993) in April 1991, 91 hospitalized patients in Cairo were reported to the Egyptian Ministry of Health with the botulism intoxication. To define the spectrum of illness and identify a food vehicle, 45 patients were interviewed and a casecontrol investigation was conducted among families of five hospitalized patients. The clinical specimens and specimens of implicated food were tested for toxin and cultured for $C$. botulinum. The hospitalized patients had symptoms consistent with botulism; 18 $(20 \%)$ of 91 reported patients died. Illness was associated with eating faseikh (uneviscerated, salted mullet fish; lower 95\% confidence limit of odds ratio $=6.6, \mathrm{P}$ $<.001)$. All 5 case-families purchased faseikh from one shop. Very high levels of type $\mathrm{E}$ botulinal toxin were detected in faseikh reported to be purchased from the implicated shop; $C$. botulinum type E was isolated from cultures of clinical specimens and from the faseikh.

Frean et al. (2002) reported that in late February 2002, two siblings aged eight and 12 years developed acute flaccid paralysis and died. Mouse bioassays revealed the presence of type A botulinum toxin in the serum of both children, and in the retrieved remains of the implicated food. The implicated vehicle of the toxin was tinned fish in tomato sauce, commercially produced in South Africa. Type A C. botulinum was cultured from the food. The most likely scenario was that corrosion damage had allowed entry of environmental organisms, including C. botulinum, to the tinned food, which was the first outbreak of human type A botulism in southern Africa to be documented, and the first fatal outbreak described; previous human cases in this region have involved type B botulinum toxin, which tends to produce milder disease. A few other outbreaks elsewhere in Africa have been

Published, the most extensive being a type E epidemic in Egypt. The commercially tinned products were not involved in any of those outbreaks.
Horowitz (2010) stated that seven known serotypes of botulism, designated A through $\mathrm{G}$; almost all human cases of botulism are caused by types A, B, and E. Botulism type $\mathrm{E}$ is the predominant serotype causing disease associated with native Arctic foods. In the circumpolar regions of the world, the coastal soils are rich in botulism type E, and consumption of fish and marine animals in these areas are the sources of clusters of botulism. Unlike spores of type A and B, botulism type $\mathrm{E}$ can withstand freezing down to $3.5^{\circ} \mathrm{C}$. Alaskan native fermentation of fish heads, fish eggs, and beaver tail allow proper an-aerobic conditions for botulinum toxin to be elaborated from C. botulinum. The con-sumption of whale meat, "muktuk" has also been associated with outbreaks of botulism in Alaska and the Canadian Arctic. Elsewhere in the Arctic regions, type $\mathrm{E}$ botulism has been associated with Norwegian "rakfisk" prepared by a process similar to fermented Alaskan foods. Outbreaks in Egypt with the salted gray mullet "faseikh", in Israel and New York linked to salted uneviscerated whitefish "kapchunka", in Iran from eating "ashbal" an un-cooked salmon, and in Japan with "izushi" the traditional fermented fish preserved in the rice have occurred. Importation of vacuumpacked whitefish from Alaska and Canada has also been associated with sporadic cases of botulism type $\mathrm{E}$ in Europe. In March 2010, the CDC released the heptavalent antitoxin (H-BAT) for use in the USA, under an Investigational New Drug program, as the preferred treatment for food-borne botulism, including type $\mathrm{E}$, which had not been covered by the bivalent antitoxin, the prior approved antitoxin product in the USA.

Mosabah and Morsy (2012) demonstrated tick paralysis for the first time in Egypt among four children living in rural area at Giza Governorate. The clinical pictures were confused with rabies; myasthensia gravis; botulism; diphtheritic polyneuropathy encountered in rural areas. But, the recovery of tick infesting the four little children and 
negative clinical and laboratory data of all diseases denoted tick paralysis. The encountered ticks infesting their farm animals were Rhipicephalus sanguineus on dogs, Hyalomma dromedarii on camels and $H$. anatolicum excavatum and Haemaphysalis sp. on goats and sheep.

Apart from Egypt, Fathalla et al. (2008) in the United Arab Emirates reported a 3month-old girl with culture-confirmed infant botulism caused by a rare double toxinproducing $C$. botulinum type $\mathrm{Ba}$. The case was not related to honey-feeding. The clinical course was prolonged, with minimal spontaneous improvement at onset, and a period of fluctuating motor weakness and nasogastric feeding dependence afterward. The neurophysiologic studies produced normal results. Human botulism immune globulin was administered empirically on day 23 of presentation, with rapid full recovery. They added that the case high-lights the importance of pursuing diagnoses of infant botulism despite normal results of neurophysiologic testing and no history of honey-feeding.

\section{Conclusion}

No doubt, botulism may be rare, but when it occurred as an outbreak, or even among a closed community like school or hospital or even in family members, it deserves the imm-

ediate medical and preventive interference.

All forms of botulism can be fatal and are considered medical emergencies. Foodborne botulism can represent a major public health threat, because there may be many other persons at risk of poisoning from eating the same contaminated food. Infant botulism can be complicated by nosocomial adverse events. The case fatality rate is less than $1 \%$ for hospitalized infants with death due to respiratory failure. Signs and symptoms of foodborne botulism include: Difficulty swallowing or speaking, dry mouth, facial weakness on both sides of face, blurred or double vision, drooping eyelids, trouble breathing, nausea, vomiting and abdominal cramps and paralysis. In wound botulism include: Difficulty swallowing or speaking, facial weakness on both sides; blurred or double vision, drooping eyelids, trouble breathing and paralysis. In infant botulism include: Constipation (first sign), floppy movements due to muscle weakness and trouble controlling head, weak cry, irritability, drooling, drooping eyelids, tiredness, difficulty sucking or feeding, and paralysis. Awareness of the botulism symptoms and the high degree of clinical suspicion is a must to make prompt diagnosis and save patient life.

\section{References}

Abrutyn, E, 1998: Botulism. In: Principles of Internal Medicine, $14^{\text {th }}$ ed, Fauci, AS, Isselbacher, KJ, Braunwald, E, et al (Eds), McGrawHill, New York.

Arnon, SS, Schechter, R, Maslanka, SE, et al, 2006: Human botulism immune globulin for the treatment of infant botulism. N. Engl. J. Med. 354:462-8.

Aureli, P, Fenicia, L, Pasolini, B, et al, 1986: Two cases of type $\mathrm{E}$ infant botulism caused by neurotoxi-genic Clostridium butyricum in Italy. J. Infect. Dis. 154:207-12.

Barnes, M, Britton, PN, Singh-Grewal, D, 2013: Of war and sausages: a case-directed review of infant botulism. J. Paediatr. Child Hlth. 49, 3:E232-4.

Bleck, TP, 2005: Clostridium botulinum (botulism). In: Principles and Practice of Infectious Diseases, $6^{\text {th }}$ ed. Mandell, GL, Bennett, JE, Dolin, R, (Eds), Churchill Livingstone, Philadelphia, PA, USA.

CDC, 1992: Outbreak of type E botulism associated with an un-eviscerated, salt-cured fish product; New Jersey, 1993. MMWR. Morb. Mortal. Wkly. Rep. 41:521-2.

Chertow, DS, Tan, ET, Maslanka, SE, et al, 2006: Botulism in 4 adults following cosmetic injections with an unlicensed, highly concentrated botulinum preparation. JAMA 296:2476.

Dowell, VR, 1984: Botulism and tetanus: selected

epidemiologic and microbiologic aspects. Rev. Infect. Dis. 6, 1:S202.

Fathalla, WM, Mohammed, KA, Ahmed, E, 2008: Infant botulism type $\mathrm{Ba}$ : first cultureconfirmed case in the United Arab Emirates. Pediatr. Neurol. 39, 3:204-6.

Frean, J, Arntzen, L, van den Heever, J, Perovic, O, 2004: Fatal type A botulism in South 
Africa, 2002. Trans. R. Soc. Trop. Med. Hyg. 98, 5:290-5.

Gottlieb, SL, Kretsinger, K, Tarkhashvili, N, et al, 2007: Long-term outcomes of 217 botulism cases in the Republic of Georgia. Clin. Infect. Dis. 45:174.

Hibbs, RG, Weber, JT, Corwin, A, Allos, BM, Abd el Rehim, MS, et al, 1996: Experience with the use of an investigational $F\left(a^{\prime}\right) 2$ heptavalent botulism immune globulin of equine origin during an outbreak of type $\mathrm{E}$ botulism in Egypt. Clin. Infect. Dis. 23, 2:337-40.

Horowitz, BZ, 2010: Type E botulism. Clin. Toxicol. (Phila). 48, 9:880-95.

Hughes, JM, Blumenthal, JR, Merson, MH, et al, 1981: Clinical features of types A and B food-borne botulism. Ann. Int. Med. 95:442-8.

Jin, R, Rummel, A, Binz, T, Brunger, AT, 2006: Botulinum neurotoxin $B$ recognizes its protein receptor with high affinity and specificity. Nature 444:1092-9.

Kongsaengdao, S, Samintarapanya, K, Rusmeechan, S, et al, 2006: An outbreak of botulism in Thailand: clinical manifestations and management of severe respiratory failure. Clin. Infect. Dis. 43:1247-52. Mechem, CC, Walter, FG, 1994: Wound Botulism. Vet. Hum. Toxicol. 36:233-9.

Mosabah, AAA, Morsy, TA, 2012: Tick paralysis: First zoonosis record in Egypt. J. Egypt. Soc. Parasitol. 42, 1:71-8.

O'Suilleabhain, P, Low, PA, Lennon, VA, 1998: Autonomic dysfunction in the LambertEaton myasthenic syndrome: Serologic and clinical correlates. Neurol. 50:88-92.

Pickett, J, Bert, B, Chaplin, E, et al, 1976: Syndrome of botulism in infancy: Clinical and electrophysiologic study. N. Engl. J. Med. 295: 770-8.

Pifko, E, Price, A, Sterner, S, 2014: Infant botulism and indications for administration of botulism immune globulin. Pediatr. Emerg. Care 30, 2:120-4.

Radšel, A, Andlovic, A, Neubauer, D, Osredkar, D, 2013: Infant botulism: first two confirmed cases in Slovenia and literature review. Eur. J. Paediatr. Neurol. 17, 6:651-6.

Reddy, P, Bleck, T, 2010: Clostridium botulin$u m$. In: Principles and Practice of Infectious
Dise- ses, $7^{\text {th }}$ ed, Mandell, G, Bennett, J, Dolin, R (eds), Churchill Livingstone Elsevier, Philadelphia.

Roblot, F, Popoff, M, Carlier, JP, et al, 2006: Botulism in patients who inhale cocaine: the first cases in France. Clin. Infect. Dis.43:e51.

Rossetto, O, Megighian, A, Scorzeto, M, Montecucco, C, 2013: Botulinum neurotoxins. Toxicon $67: 31-6$

Santos, JI, Swenson, P, Glasgow, LA, 1981: Potentiation of Clostridium botulinum toxin by aminoglycoside antibiotics: Clinical and laboratory observations. Pediatrics 68:50-8.

Schreiner, MS, Field, E, Ruddy, R, 1991: Infant botulism: a review of 12 years' experience at the Children's Hospital of Philadelphia. Pediatrics 87:159-64.

Sellin, LC, 1984: Botulism-an update. Mil. Med. 149: 12.

Sheth, AN, Wiersma, P, Atrubin, D, et al, 2008: International outbreak of severe botulism with pro-longed toxemia caused by commercial carrot juice. Clin Infect Dis. 47:1245.

Simpson, L, 2013: The life history of a botulinum toxin molecule. Toxicon_68:40-59.

Sobel, J, Malavet, M, John, S, 2007: Outbreak of clinically mild botulism type E illness from home-salted fish in patients presenting with predominantly gastrointestinal symptoms. Clin Infect Dis. 45:e14

Tacket, CO, Shandera, WX, Mann, JM, et al, 1984: Equine antitoxin use and other factors that produce outcomes in type A food borne botulism. Am. J. Med. 76:794.

Townes, JM, Cieslak, PR, Hatheway, CL, et al, 1996: An outbreak of type A botulism associated with a commercial cheese sauce. Ann Intern Med.125:558.

Weber, JT, Hibbs, RG Jr, Darwish, A, Mishu, B, Corwin, AL, et al, 1993: A massive outbreak of type E botulism associated with traditional salted fish in Cairo. J. Infect. Dis. 167, 2:451-4.

Werner, SB, Passaro, D, McGee, J, et al, 2000: Wound botulism in California, 19511998: recent epidemic in heroin injectors. Clin. Infect. Dis. 31:101-8. 